\title{
Self-consistent formation of a 0.5 cyclotron frequency gap in magnetospheric whistler- mode waves
}

Article

Published Version

Ratcliffe, H. and Watt, C. E. J. (2017) Self-consistent formation of a 0.5 cyclotron frequency gap in magnetospheric whistlermode waves. Journal of Geophysical Research - Space Physics, 122 (8). pp. 8166-8180. ISSN 0148-0227 doi: https://doi.org/10.1002/2017JA024399 Available at https://centaur.reading.ac.uk/71971/

It is advisable to refer to the publisher's version if you intend to cite from the work. See Guidance on citing.

To link to this article DOI: http://dx.doi.org/10.1002/2017JA024399

Publisher: American Geophysical Union

All outputs in CentAUR are protected by Intellectual Property Rights law, including copyright law. Copyright and IPR is retained by the creators or other copyright holders. Terms and conditions for use of this material are defined in the End User Agreement.

www.reading.ac.uk/centaur 
Central Archive at the University of Reading

Reading's research outputs online 


\section{Journal of Geophysical Research: Space Physics}

\section{RESEARCH ARTICLE \\ 10.1002/2017JA024399 \\ Self-consistent formation of a 0.5 cyclotron frequency gap in magnetospheric whistler mode waves}

Key Points:

- The common whistler mode

frequency gap can be explained

self-consistently using kinetic plasma theory

- New, detailed simulations are presented exploring the mechanism across a range of parameters

- Electron distribution functions

remodel locally and rapidly, so linear growth theory does not predict these effects from initial conditions

Correspondence to:

H. Ratcliffe,

h.ratcliffe@warwick.ac.uk

Citation:

Ratcliffe, H. and C. E. J. Watt (2017), Self-consistent formation of a 0.5 cyclotron frequency gap in magnetospheric whistler mode waves, J. Geophys. Res. Space Physics, 122, doi:10.1002/2017JA024399.

Received 25 MAY 2017 Accepted 19 JUL 2017

Accepted article online 25 JUL 2017

(O2017. American Geophysical Union. All Rights Reserved.

\author{
H. Ratcliffe ${ }^{1}$ (D) and C. E. J. Watt ${ }^{1}$ iD \\ ${ }^{1}$ Department of Meteorology, University of Reading, Reading, UK
}

Abstract Decades of in situ observations of whistler mode waves in Earth's magnetosphere reveal that there is frequently a gap in the spectral power at around half the local electron gyrofrequency. Recent theoretical and kinetic simulation studies have suggested that the gap arises due to the presence of temperature anisotropy in both a "warm" and a "hot" electron population, leading to two separate (and independent) regions of wave growth in frequency space. We present two-dimensional kinetic plasma simulations using the powerful EPOCH (Extendable PIC Open Collaboration) particle-in-cell code that offer an alternative explanation. After an initial linear-growth period, our simulations show self-consistent formation of a gap feature. In most cases this arises where linear theory predicts the maximum growth rate and is associated with subtle local structuring of the hot electron distributions. This feature persists in multiple simulations with varying hot electron parameters. We discuss these results in the context of in situ observations of both waves and electron distribution functions and argue that the rapid reorganization of electron distributions in a small, but key, region of phase space during the growth of whistler mode waves naturally results in the spectral gap often observed at half the electron gyrofrequency.

\section{Introduction}

Whistler mode chorus waves are found throughout low-density regions in the magnetosphere outside of the plasmasphere [e.g., Burtis and Helliwell, 1969; Meredith et al., 2001; Li et al., 2011 b; Spasojevic and Inan, 2010; Sigsbee et al., 2010; Bunch et al., 2011; Agapitov et al., 2013; Meredith et al., 2012; Bunch et al., 2012]. They are important for the acceleration and pitch angle scattering of high-energy electrons in Earth's outer radiation belt [Horne et al., 2005; Ni et al., 2011a; Thorne et al., 2013] and are responsible for the diffuse aurora [Thorne et al., 2010; Nishimura et al., 2013].

A gap in the chorus mode at around half the electron gyrofrequency $\omega_{\text {ce }}$ is a common feature in whistler mode spectra [Burtis and Helliwell, 1969; Santolik et al., 2004; Santolik et al., 2008; Taubenschuss et al., 2014], although this feature is not seen in every case [Burtis and Helliwell, 1976; Cornilleau-Wehrlin et al., 1978; Kurita et al., 2012; Taubenschuss et al., 2015]. A spectral gap at near $\omega_{\text {ce }} / 2$ has also been observed in whistler mode emissions at other planets [Coroniti et al., 1984]. Waves display a banded structure with "lower band" occupying $\omega_{\mathrm{lh}}<\omega<0.5 \omega_{\mathrm{ce}}$ [Tsurutani and Smith, 1977], where $\omega_{\mathrm{lh}}$ is the lower-hybrid frequency, and "upper band" occupying $0.5 \omega_{\mathrm{ce}}<\omega<\omega_{\mathrm{ce}}$. Several explanations have been proposed for the banded emissions that either require the independent generation of each band or invoke the preferential absorption of waves at different frequencies across the whistler mode band. For example, Bell et al. [2009] postulate that lower band chorus is generated within enhanced density ducts and upper band chorus is generated within depleted ducts. Liu et al. [2011] and Fu et al. [2014] investigate the energetic electrons that provide the source of free energy for the waves, and argue that distinct anisotropic warm $(<1 \mathrm{keV})$ and hot ( $>10 \mathrm{keV})$ components independently provide the upper and lower band whistler mode emissions, respectively. Bortnik et al. [2006] showed that convective Landau damping was theoretically reduced immediately above $\omega_{\text {ce }} / 2$ and that this could contribute to the appearance of suppressed emissions at half the gyrofrequency, but the effect only became apparent after the chorus had propagated a significant distance away from the magnetic equator. The suppression of waves close to half the gyrofrequency is suggested by Omura et al. [2008, 2009], who argue that as whistler mode waves propagate along the inhomogeneous magnetic field of the magnetosphere, small deviations from perfectly field-aligned wave normals result in nonlinear damping of waves with frequencies close to $\omega_{\text {ce }} / 2$. 
Although distinct warm and hot plasma populations can neatly predict the presence of distinct upper and lower bands of whistler mode waves, these theories rely upon particular choices of plasma parameters to ensure a gap at the correct frequency. We show in this paper that the linear theory predictions of growth for Bi-Maxwellian plasma are very sensitively dependent upon the temperature and anisotropy of each component. Given the common occurrence of a $\omega_{\text {ce }} / 2$ gap in the whistler mode spectrum, and the range of possible temperatures and anisotropies possible in the magnetosphere, we investigate further the behavior of whistler mode waves given a range of plasma conditions. We perform numerical experiments using a particle-in-cell code to demonstrate that the rapid nonlinear evolution in velocity space of the anisotropic electron distribution function in the presence of growing whistler mode waves naturally results in a spectral gap at $\omega_{\text {ce }} / 2$ long before any electron holes develop in phase space and nonlinear growth starts [Omura et al., 2008, 2009]. We also discuss the minimum requirements for numerical experiments to demonstrate the self-consistent formation of the frequency gap.

In section 2 we present important theoretical results pertaining to the growth of whistler mode waves. The particle-in-cell code EPOCH (Extendable PIC Open Collaboration) used throughout the paper, is presented in section 3, along with a description of the postsimulation analysis we have used to study the wave-particle interactions. We present our results in section 4, followed by a discussion and summary in section 5 .

\section{Theoretical Considerations}

The growth $\gamma$ of small amplitude electromagnetic waves can be estimated from linear theory

$$
\gamma=-D_{l}(\omega)\left[\frac{\partial D_{R}(\omega)}{\partial \omega}\right]^{-1}
$$

where $D_{R}$ is the real part and $D_{l}$ is the imaginary part of the full relativistic kinetic dispersion relation [Treumann and Baumjohann, 1997]. Applying equation (1) to a single hot Bi-Maxwellian particle distribution function with temperature anisotropy predicts growth that is roughly Gaussian-shaped with respect to frequency. Previous plasma models of the inner magnetosphere indicate that more than one Bi-Maxwellian component is often required to model the energetic plasma with components at different average temperatures [Denton et al., 2010; Watt et al., 2012]. It is important to note that a wide range of combinations of plasma parameters are possible at different times.

Combining a pair of heated plasma distributions can produce two distinct growth peaks, and careful tuning of the plasma parameters can then produce a gap feature at around $0.5 \omega_{\text {ce }}$ [e.g., Fu et al., 2014]. Figure 1 shows the growth rates calculated using equation (1) for a range of different plasma parameters. The black line indicates the growth possible for parameters chosen by Fu et al. [2014] which we will denote as M0. The other colored lines indicate growth rates for the numerical experiments contained in this paper and listed in Table 1: M1 is shown in purple, M2 in blue, M3 in green, M4 in orange, and M5 in red. Different choices of warm and hot populations can provide a range of different growth behavior: set M3 (green line), for example, provides a peak in growth exactly where there is a local minimum in growth for set M0 (black line). In this paper we will investigate the evolution of wave growth for all of these sets of parameters using two-dimensional fully self-consistent particle-in-cell simulations.

When initiating studies of wave growth, the choice of parameters can be challenging. Observations show that the particle distributions outside the plasmasphere are often strongly non-Maxwellian and must be rapidly evolving from an unstable injected state to the stable "pancake" distribution [Wrenn et al., 1979; Meredith et al., 1999; Gannon et al., 2007; Li et al., 2011c; Tao et al., 2011]. The pancake distribution [Meredith et al., 1999] is the distribution which is perfectly stable against whistler mode wave growth and likely indicates the previous action of whistler mode waves to reduce the free energy in an unstable anisotropic distribution. For a pancake distribution, phase space density is constant along lines given by

$$
\begin{aligned}
& v_{\perp}=\sqrt{\frac{2 E_{c}}{m_{e}}\left[f\left(x_{c}\right)-f(x)\right]} \\
& v_{\|}=\sqrt{\frac{2 E_{c}}{m_{e}}} g(x)
\end{aligned}
$$




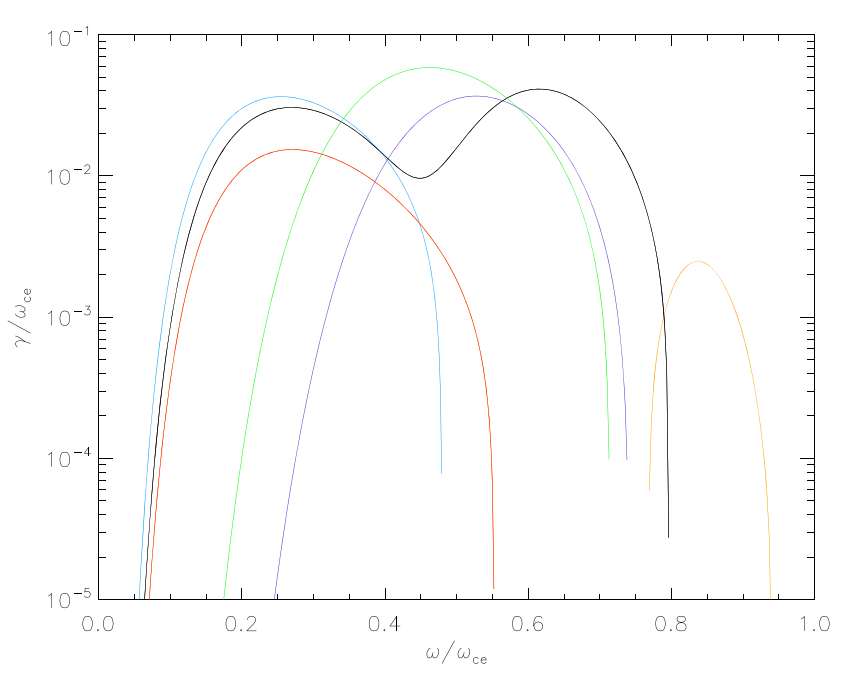

Figure 1. Whistler mode growth rates for parallel wave vector for six sets of parameters including those of Fu et al. [2014] (black line). Referring to Table 1 which lists the full set of parameters, set $M 1$ is shown in purple, M2 in blue, M3 in green, M4 in orange, and M5 in red. Note that the orange line is hidden under the red on the left. Hot electron thermal velocities range from 0.075 to $0.5 c$, warm electron from 0.015 to $0.075 \mathrm{c}$ with densities from 0.01 to 0.18 times the background.

with

$$
\begin{aligned}
& f(x)=\left[\left(\frac{1-x}{x}\right)+2 x-\ln x\right] \\
& g(x)=-\sqrt{\frac{(1-x)^{3}}{x}}
\end{aligned}
$$

and $x=\omega / \omega_{\mathrm{ce}}$. The parameter $x_{c}$ is the value of $x$ at pitch angle zero and $E_{c}=\frac{1}{2} m_{e} c^{2}\left(\omega_{\mathrm{ce}} / \omega_{\mathrm{pe}}\right)^{2}$ the magnetic energy per particle. However, the model given by equation (2) cannot dictate the spacing between isolines, that is, the lines of constant $x_{c}$ and so does not fully specify a distribution. We cannot predict the final stable distribution from the initial Maxwellian, but we expect evolution toward these contours. For our simulations, we must therefore choose an initial electron distribution function that is unstable (e.g., Bi-Maxwellian) in order to observe the growth of the waves, rather than using observed electron distribution functions that are more likely to be stable to wave growth.

\section{Methods}

We perform fully self-consistent particle-in-cell simulations for a range of plasma parameters. Kinetic descriptions of the plasma are necessary because of the waves' resonant interaction with electrons, satisfying:

$$
\omega-k_{\|} v_{\|}=\omega_{n}, \omega_{n}:=s n \omega_{\text {ce }} / \gamma
$$

where $s= \pm 1, n$ is an integer, $\gamma$ is the relativistic gamma factor, and $\omega_{\text {ce }}=|q| B / m_{e}$ is the (unsigned) local electron cyclotron frequency.

Table 1. Run Parameters for Our Five Simulations ${ }^{\mathrm{a}}$

\begin{tabular}{lcccccccc}
\multicolumn{9}{c}{ Hot } \\
\hline Label & $v_{x} / c$ & $v_{y} / c$ & $\mathrm{~A}$ & $n_{h} / n_{e}$ & $v_{x} / c$ & $v_{y} / c$ & $\mathrm{~A}$ & $n_{w} / n_{e}$ \\
M1 & 0.075 & 0.15 & 3.0 & 0.17 & 0.015 & 0.038 & 5.4 & 0.17 \\
M2 & 0.225 & 0.32 & 1.0 & 0.15 & 0.015 & 0.038 & 5.4 & 0.15 \\
M3 & 0.1 & 0.2 & 3.0 & 0.17 & 0.015 & 0.038 & 5.4 & 0.18 \\
M4 & 0.225 & 0.5 & 4.0 & 0.01 & 0.015 & 0.075 & 24 & 0.01 \\
M5 & 0.225 & 0.5 & 4.0 & 0.01 & 0.015 & 0.038 & 5.4 & 0.01 \\
\hline
\end{tabular}

${ }^{a}$ We show the hot and warm components separately and list the $x$ and $y$ thermal speeds, the resulting anisotropy, and the density of the component as fraction of the background density. 
The number of phase space dimensions necessary to simulate the interaction between whistler mode waves and electrons is an important consideration. Theoretically, for a single anisotropic electron distribution, the maximum growth rate of the whistler mode waves is field aligned, with finite, but smaller, growth for small oblique angles [e.g., Watt et al., 2011]. However, observational studies have shown that whistler mode wave normal angles in the magnetosphere can have a range of values from close to the field-aligned direction, to large oblique angles [Haque et al., 2010; Artemyev et al., 2012; Agapitov et al., 2013; Li et al., 2012, 2013; Taubenschuss et al., 2014, 2015; Artemyev et al., 2015; Hartley et al., 2016; Li et al., 2016; Taubenschuss et al., 2016; Gao et al., 2016; Artemyev et al., 2016]. We choose to perform two-dimensional (2-D) simulations in order to include important oblique wave vectors. To perform 2-D simulations with sufficient particles to resolve the fairly weak wave $E$ and $B$ fields requires significant computing time. Computations were performed on the Data Analytic (Cambridge) and Data Centric (Durham) systems of the STFC DiRAC HPC Facility. Each large numerical experiment M1-M5 (see parameters in Table 1) required approximately $12.5 \mathrm{k}$ core hours. Storage and $1 \mathrm{O}$ limitations restricted the output to 4-5 spectra and distribution functions per run. We therefore also show results from a number of smaller numerical experiments with the same parameters as M1 - M5 performed on a 16-core workstation. In these, particle number and box size were reduced to obtain a shorter simulation time. The combination of results from each type of numerical experiment has provided the required insight and will be used to shape future sets of numerical experiments.

\subsection{Code Description and Setup}

We use EPOCH2D [Arber et al., 2015], a Birdsall and Langdon-type PIC code [Birdsall and Langdon, 2004] using Villasenor and Buneman current deposition [Villasenor and Buneman, 1992]. EPOCH solves Maxwell's equations combined with the equations of motion for charged particles in an EM field to provide a direct simulation of collisionless plasma. The electron-proton mass ratio is set to the real value of 1:1836.2. EPOCH uses a Cartesian grid, which in 2-D means all quantities may vary in the $x$ and $y$ directions. In the $z$ direction, plasma quantities are assumed constant but are well defined: for example, the plasma components have a well-defined $z$ direction temperature. All quantities are thus given in normal SI units. In what follows, we set an ambient magnetic field along the $x$ direction, so refer to $x$ as parallel, $y$ and $z$ as perpendicular, and assume symmetry such that in a 3-D simulation the $z$ direction behavior would follow $y$.

We assume cold, fully ionized hydrogen background plasma with temperature $10^{4} \mathrm{~K}$ in both parallel and perpendicular directions, with an ambient magnetic field along the $x$ direction of $300 \mathrm{nT}$. The plasma frequency is $\omega_{\text {pe }}=3 \omega_{\text {ce }}$, i.e., a background plasma density of $n_{e}=7.9 \times 10^{6} \mathrm{~m}^{-3}$. These parameters are chosen to represent plasma conditions near the equator at a magnetospheric location of $L \sim 5$.

To initiate a situation unstable to the growth of whistler mode waves, we then add two additional electron components, denoted "warm" and "hot." Both components are Bi-Maxwellian with larger perpendicular than parallel temperature. Initial particle distributions for each component are Bi-Maxwellians of the form

$$
f\left(p_{\|}, p_{\perp}\right)=C \exp \left(-\frac{p_{\|}^{2}}{p_{\mathrm{th}, \|}^{2}}\right) \exp \left(-\frac{p_{\perp}^{2}}{p_{\mathrm{th}, \perp}^{2}}\right)
$$

where $p_{\mathrm{th}, \|}$ and $p_{\mathrm{th}, \perp}$ are the parallel and perpendicular thermal momenta, and $C$ is the normalization constant. The distributions are normalized so that

$$
\int f\left(p_{\|}, p_{\perp}\right) \mathrm{d} p_{\|} \mathrm{d}^{2} p_{\perp}=n_{e}
$$

in units of $\mathrm{m}^{-3}$. Momentum-space distributions are averaged over the spatial domain for output.

Throughout this paper, plasma anisotropy is defined as

$$
A=\frac{T_{\perp}}{T_{\|}}-1=\frac{2 v_{\mathrm{th}, y}^{2}}{v_{\mathrm{th}, x}^{2}}-1
$$

where $T_{\perp}$ and $T_{\|}$are the perpendicular and parallel temperatures, respectively, and $v_{\mathrm{th}, x}$ and $v_{\mathrm{th}, y}$ are the thermal speeds in the $x$ and $y$ directions, respectively. Note that the $z$ direction thermal speed is the same as the $y$ direction speed and so $v_{\mathrm{th}, \perp}^{2}=v_{\mathrm{th}, y}^{2}+v_{\mathrm{th}, z}^{2}=2 v_{\mathrm{th}, y}^{2}$. Our definition of $A$ ensures that an isotropic distribution would have an anisotropy of zero. The spatial resolution is $0.06 c / \omega_{\text {ce }}$ in both $x$ and $y$ directions, with 1024 and 512 grid points, respectively. This gives at least three cycles over the box length and three points per cycle in both directions for wave numbers between $k=2.0 \times 10^{-5} \mathrm{~m}^{-1}$ and $k=0.001 \mathrm{~m}^{-1}$. Periodic boundary 


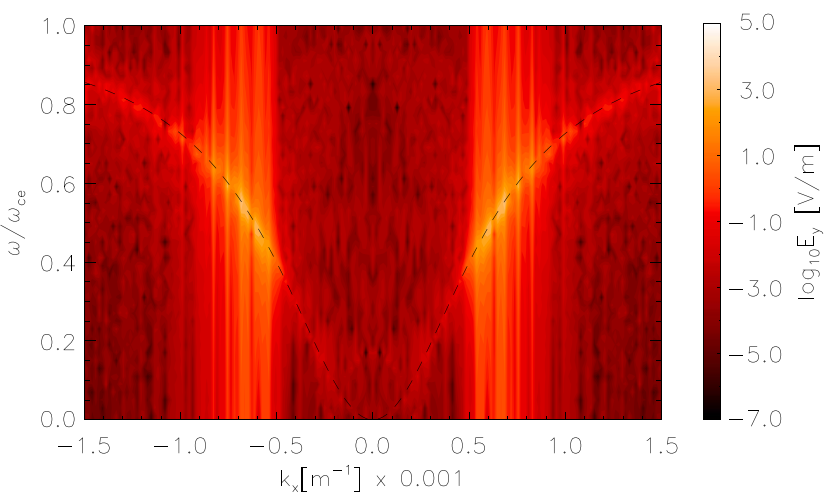

Figure 2. Example of the Fourier transformed $E_{y}$ component of electric field against $k_{x}$ and $\omega$ for $k_{y}=0$ (propagation parallel to the ambient magnetic field) over the initial growth period. The dashed line is the theoretical whistler dispersion, as given by equation (8). conditions are used in both directions. We use between 50 and 500 particles per cell ( $p p c)$ for each species (ions, background electrons, warm, and hot electrons, respectively) denoted $\mathrm{ppc} / \mathrm{s}$. The large simulations (M1-M5) use $500 \mathrm{ppc} / \mathrm{s}$, while 50, 150, and 300 $\mathrm{ppc} / \mathrm{s}$ simulations are also used for convergence testing (see section 4.2). As discussed above, the large simulations require supercomputing time, and so some additional, smaller experiments were performed to obtain more detailed distribution functions, spectra over intermediate times, and to perform some additional investigations. These less computationally expensive numerical experiments use a smaller box and lower particle number, 512 by 256 cells with resolution as above and $150 \mathrm{ppc} / \mathrm{s}$, respectively. Detailed convergence testing is discussed below in section 4.2, but we note that between 150 and $500 \mathrm{ppc} / \mathrm{s}$ the quantities of interest here (e.g., spectra, growth rates, particle distributions) show changes of generally less than $10 \%$.

\subsection{Whistler Mode Wave Analysis}

To extract wave spectra from the $E$ and $B$ fields, and to discriminate between forward and backward waves, we perform Fourier analysis in space and time together. The FFT of a given field is typically performed using 500 time bins and the entire spatial domain, with an example of the results for simulation M1 shown in Figure 2. Wave spectra of the form $\tilde{E}(\omega, \theta)$, with $\theta$ the wave normal angle, are then extracted by summing the wave power in a band around the dispersion curve, given by

$$
\omega=\frac{-c^{2} k^{2} \omega_{c e} \cos \theta}{c^{2} k^{2}+\omega_{p e}^{2}}
$$

where $\omega$ is the (real) wave frequency, $c$ is the speed of light, $k$ is the magnitude of the wave vector, $\theta$ is the angle between the wave vector and the ambient magnetic field, and $\omega_{p e}$ is the plasma frequency. A spread of $10 \%$ in local frequency was used to reduce the contributions of PIC noise while capturing all whistler mode power. The resulting $\tilde{E}(\omega, \theta)$ is then measured in $\mathrm{V} / \mathrm{m}$, with $\tilde{B}(\omega, \theta)$ in $T$. The total wave power is given by the sum of the power at each frequency in the spectrum.

To derive growth rates we assume linear growth over a given interval, i.e.,

$$
\frac{\mathrm{d} E_{k}}{\mathrm{~d} t}=\gamma_{k} E_{k}
$$

with $\gamma$ a constant for each value of wave number $k$ and so

$$
\gamma=0.5 \ln \left(\frac{E\left(t_{2}\right)^{2}}{E\left(t_{1}\right)^{2}}\right) /\left(t_{2}-t_{1}\right)
$$

over the time interval $\left[t_{1}, t_{2}\right]$. The time FFTs for spectrum extraction require at least three periods of the lowest frequency expected. For frequencies down to about $0.1 \omega_{\text {ce, }}$ which is where we see significant power, this means 30 inverse cyclotron periods minimum, so we cannot evaluate the spectrum at exactly $t=0$. Instead, we estimate the "initial" noise using the power in the wings $(\omega \sim 0.9)$ of the first spectrum.

Predictions of growth rates are calculated directly from EPOCH distribution functions at different stages of simulation evolution using equations (23)-(25) of Xiao et al. [1998]. The integrals are evaluated numerically using the trapezium rule. To evaluate the necessary derivatives of the distribution functions, we use a simple one-sided difference, with fixed small $\mathrm{d} p$ for analytic distributions. When calculating growth rates from EPOCH distribution function output, we smooth in phase space to reduce noise due to the discrete simulation pseudo-particles. For this, we use a Gaussian smoothing on the 2-D $p_{x} p_{y}$ distribution functions. In the 


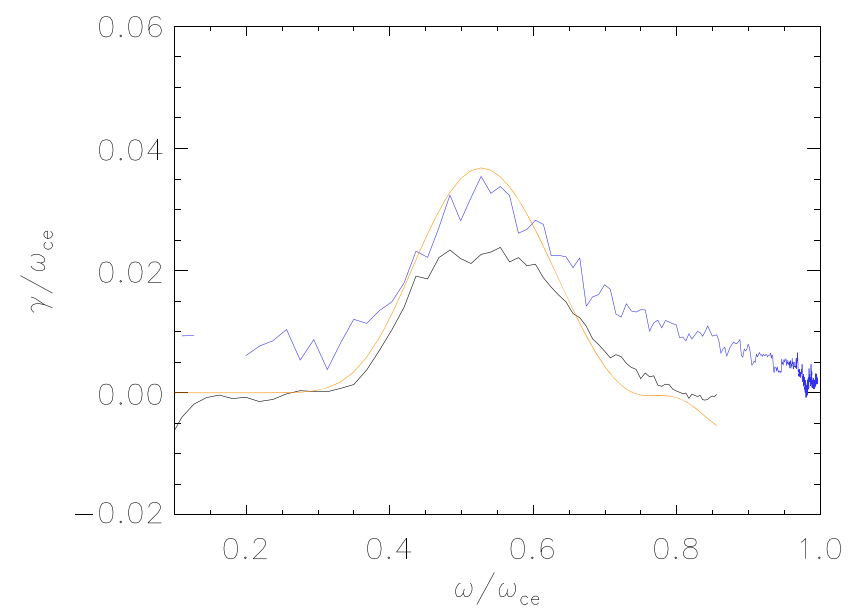

Figure 3. Initial wave growth rates in a typical simulation. The black line is the growth rate derived from the simulation $E_{y}$ component over the first $0.006 \mathrm{~s}$ derived as described in the previous section and summed over all angles. The blue line is the same construction using only the first $0.003 \mathrm{~s}$. The red line shows the analytic prediction of growth rate using the equations of Xiao et al. [1998].

equation (7) of $A=5.4$, with a density of $n_{w}=0.18 n_{e}$. The "hot" component has $v_{\text {" }}=0.075 c\left(T_{1}=1.6 \mathrm{keV}\right)$ $v_{\mathrm{th}, \perp}=\sqrt{2} v_{\mathrm{th}, y}=0.21 \mathrm{c}\left(T_{\perp}=11.5 \mathrm{keV}\right), A=3.0$ and $n_{h}=0.17 n_{e}$.

The initial growth of waves, shown in Figure 2, follows the linear theory prediction very well. We see growth of a single peak centered on $0.5 \omega_{\mathrm{ce}}$, produced by the hot plasma component. This stage lasts around $\sim 0.01 \mathrm{~s}$ and its derived growth rate is shown in Figure 3, along with the linear theory growth rate prediction. The Fourier analysis described in section 3.2 necessitates taking the first 50 cyclotron periods ( $0.006 \mathrm{~s}$ ) for a reasonably clean spectrum, during which the wave-particle interaction changes significantly. Growth near $0.5 \omega_{\text {ce }}$ is slightly less than the initial linear prediction, whereas growth for $\omega<0.45 \omega_{\text {ce }}$ and for $\omega>0.6 \omega_{\text {ce }}$ matches the initial linear predictions very well. A spectrum calculated using only the first half of this data period is also shown, which although rather noisy, agrees very well with the linear prediction of growth rate at $\omega=0.5 \omega_{\text {ce }}$. We will discuss the rapid evolution of this instability in further detail below.

In the initial stages of the simulation, the distribution function of the hot component begins to form into the typical "pancake" shape (see equation (2)), starting first at $p \sim 0.1-0.3 \mathrm{c}$ before proceeding to higher and lower momenta as shown in Figure 4. Simultaneously, the distribution isotropizes, and so it takes longer to establish the full pancake shape at larger momenta. The warm component (not shown), initially evolves toward a pancake, but by $0.048 \mathrm{~s}$ it is almost completely isotropized, taking some energy from the hot distribution in the process. The total electron energy is conserved to better than $5 \%$ over the simulation.

Toward the end of this first phase, we observe the formation of a "kink" or shoulder feature in the hot plasma parallel distribution function, $f\left(p_{x}\right)=\int \mathrm{d} p_{y} f\left(p_{x}, p_{y}\right)$, shown in Figure 5. It is a reduction in the gradient of the distribution function which would appear as a plateau if it were more pronounced. We show the gradient of the distribution function in Figure 5 to highlight this change. From the Doppler resonance condition of equation (4), for the primary resonance at $n=-1$, we calculate that the center of the kink at $0.15 \mathrm{c}$ corresponds

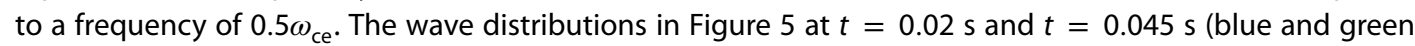
lines, respectively) display a clear dropout at precisely this frequency. The simulation domain is periodic, so we cannot distinguish the Landau and $n=-1$ resonances at $0.5 \omega_{\text {ce }}$ by direction [Artemyev et al., 2016]. However, we show later (section 4.4) another simulation with different initial parameters, where the $n=-1$ case can be identified with the same behavior.

As the simulation continues, the distribution function of the hot plasma component becomes more pancake shaped, the kink deepens, and, finally smooths out. The wave spectra show a deepening dropout at $0.5 \omega_{\text {ce }}$ and an increasing absorption over the entire upper band. By $0.07 \mathrm{~s}$ the upper band has returned to noise, driven by absorption in the increasingly isotropic warm plasma component. 

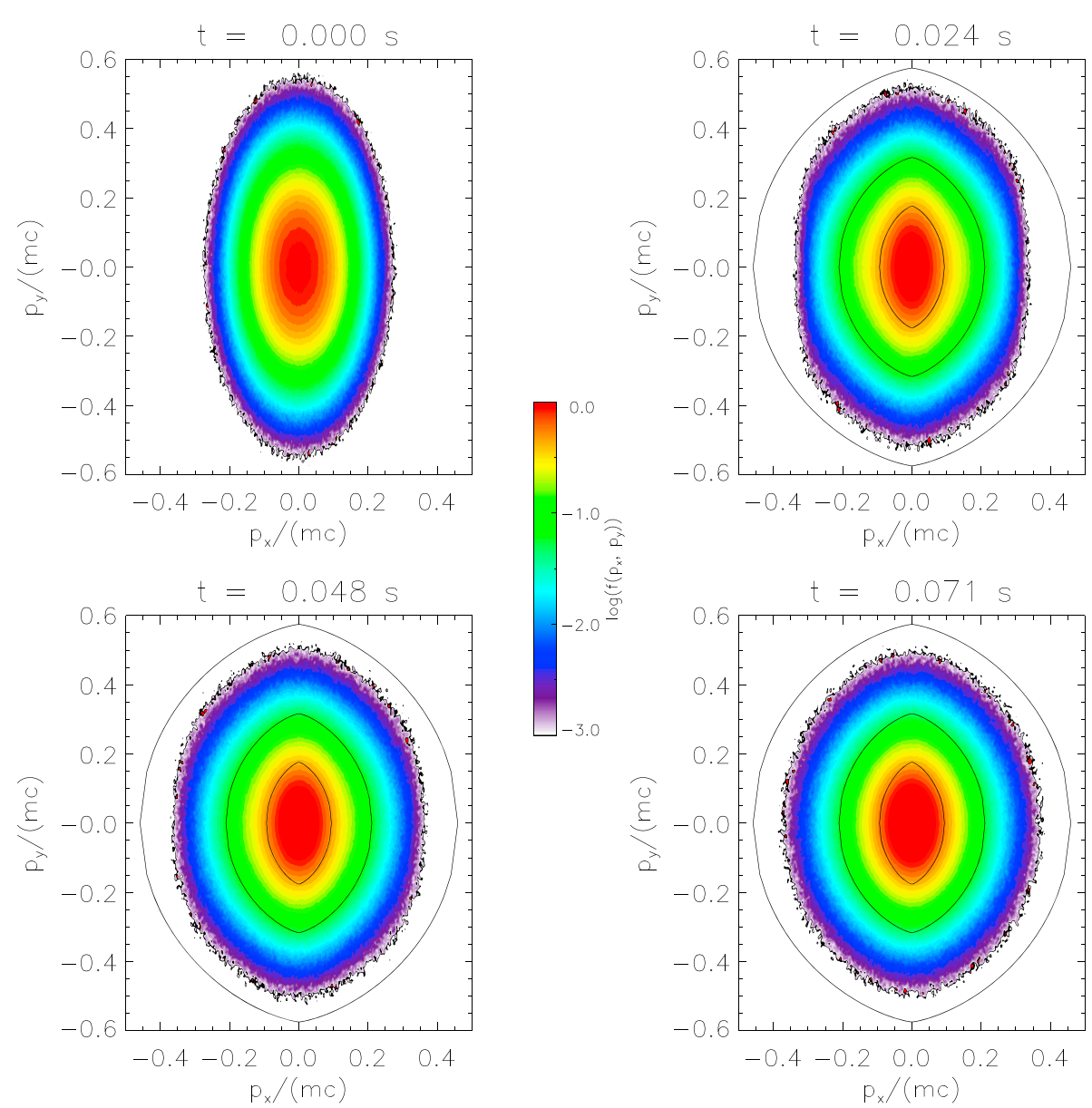

Figure 4. Initial evolution of the hot plasma distribution function for the typical simulation. The solid lines show the isolines of a pancake distribution for $x_{c}$ of $0.2,0.4,0.6$. Note that this plot shows results from an additional, lower ppc (150/cell), simulation, outputting distributions in more detail than was possible with the main case.

There is good evidence that the strong initial wave growth around $0.5 \omega_{\text {ce }}$ causes this kink in the distribution function. It is both colocated in velocity/frequency space, and contemporary in time. The kink appears shortly after the initial growth period. Even by $0.006 \mathrm{~s}$ it is clear that particles are absorbing, rather than producing, waves around $0.5 \omega_{\mathrm{ce}}$, as we see clear shortfall in wave levels in Figure 3 . The shoulder feature persists throughout the simulation, although by $0.07 \mathrm{~s}$ it is significantly reduced, by which time there are very few or no waves around $0.5 \omega_{\mathrm{ce}}$.
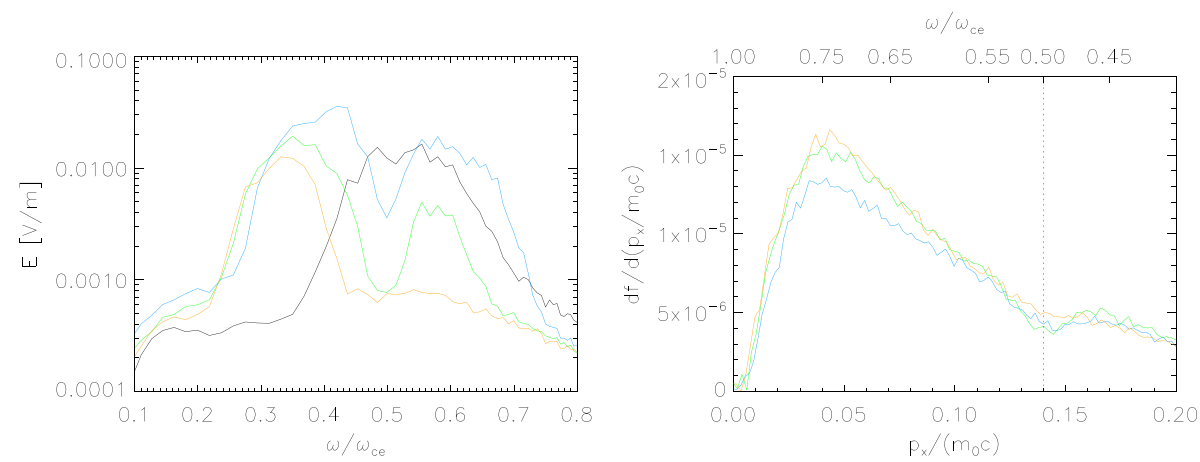

Figure 5. Wave spectra and hot plasma parallel distribution derivative during the typical simulation. The colors correspond to times centered on $t=0.003 \mathrm{~s}$ (black line), $t=0.02 \mathrm{~s}$ (blue), $t=0.05 \mathrm{~s}$ (green), and $t=0.07 \mathrm{~s}$ (orange). 


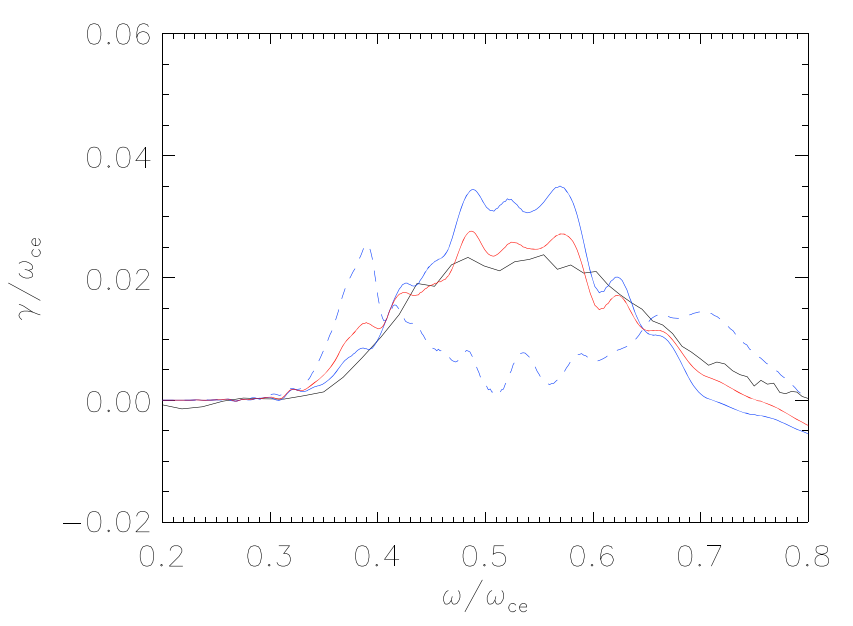

Figure 6. Wave growth rates for the typical simulation compared with predictions obtained from the simulation distribution functions. The black line is the growth rate obtained from the evolution of the electric field over $t=0 \mathrm{~s}$ to $t=0.006 \mathrm{~s}$. The linear theory predictions use the hot component distribution function only and are shown for $t=0.0 \mathrm{~s}$ (blue solid line) and $t=0.006 \mathrm{~s}$ (blue dashed line) and a 3:1 weighted average of these (red).

While the derivative never crosses zero, i.e., this is not a true inflection of the distribution, it does represent a flattening of the expected gradient. The net effect of the waves is to transfer energy from the slower electrons below the inflection to the faster ones on its upper side. Given the resonance condition, equation (4), this amounts to a transfer of energy from waves at higher frequencies to waves at lower frequencies. We emphasize that it is not necessary that the waves at any instant contain energy comparable to the electrons here, only that they can act as an energy transfer mechanism. The net absorption of waves in creating the kink is what causes the visible dropout. We discuss this proposal in section 4 in context of our full set of simulations.

Given the modification of the distributions, we do not expect the Maxwellian-based linear theory prediction of growth to hold for longer than the very first stage of the simulation, $0.01 \mathrm{~s}$ in this case. Instead, the linear equation for calculating growth can be applied directly to the observed EPOCH distribution functions after normalizing and smoothing (as described in section 3 ). The growth rates thus predicted agree very well in the initial part of the simulation as shown in Figure 6 . Since the growth rate calculation from the wave spectrum uses data over the first $0.006 \mathrm{~s}$, we show the linear growth rate prediction based on the distribution functions at both the beginning and end of this period and a weighted average of the two. The weighted average of the predictions agrees remarkably well with the observed wave levels and provides strong evidence that we have identified the source of the discrepancy noted in Figure 3, where growth is reduced around $0.5 \omega_{\mathrm{ce}}$.

Figure 7 shows the predicted growth rates over time with and without the warm plasma component influence. We see a clear absorptive effect above $0.7 \omega_{c e}$ and spreading downward as the simulation proceeds.
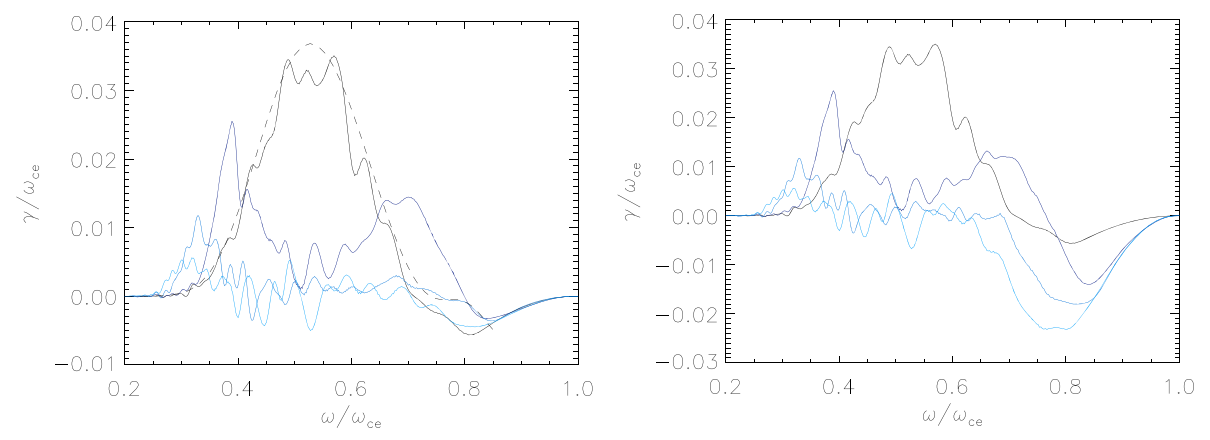

Figure 7. Growth rates as predicted by linear theory for the actual simulation particle distributions over time during the early part of the simulation. The dashed line is for the input Maxwellian. (left) Hot Maxwellian component only. (right) Hot and warm components. The times, from dark to light blue, are $0.0 \mathrm{~s}$ (black), $0.006 \mathrm{~s}$ (darkest blue), $0.012 \mathrm{~s}$ (mid blue), $0.018 \mathrm{~s}$ (lighter blue). 


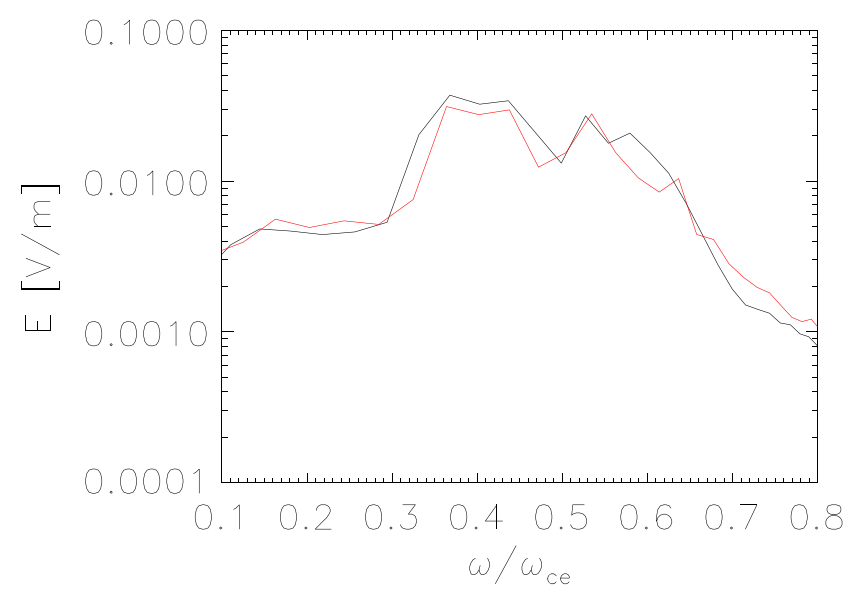

Figure 8. The contribution of the warm plasma component to growth at $t=0.018 \mathrm{~s}$. The black line is including, the red excluding the warm plasma. Note that these simulations use $150 \mathrm{ppc}$ and a smaller box due to computational constraints.

above the true thermal wave background, stronger absorption will not be reproduced. The earlier time shows clear growth at around $\omega=0.2$ to $0.45 \omega_{\text {ce, }}$, which is also seen in the prediction. The later time shows almost entirely absorption, except a small band at $0.3 \omega_{\text {ce }}$, again matching the evolving linear predictions well. Above about $0.4 \omega_{\text {ce }}$ the prediction is for strong absorption, which cannot be reproduced in the PIC simulation for these levels of $\mathrm{ppc} / \mathrm{s}$, but a weak absorption is still present.

\subsection{Convergence Testing}

Before proceeding further, we discuss the convergence of the simulations as the $\mathrm{ppc} / \mathrm{s}$ levels are increased. At $50 \mathrm{ppc} / \mathrm{s}$ the growing waves never exceed noise level. Figure 10 shows the initial wave growth rates for 150 and $500 \mathrm{ppc} / \mathrm{s}$. The simulation with $150 \mathrm{ppc} / \mathrm{s}$ shows significantly more noise at low and high frequencies $\left(\omega<0.35 \omega_{\text {ce }}\right.$ and $\left.\omega>0.7 \omega_{\text {ce }}\right)$, but the peak growth rate values are very similar. Over this range, the average values are $\gamma / \omega_{\text {ce }}=0.017$ (500 ppc/s) and 0.019 (150 ppc/s), i.e., a change of less than $20 \%$, while the value at $0.5 \omega_{\text {ce }}$ changes by less than $10 \%$. Figure 10 shows the wave spectra for 2 times and both ppc levels. The lower ppc run shows an increased wave level initially both due to the slightly enhanced growth rate and to the higher noise baseline. At later times, both show a clear gap formation and twin-peaked structure.

We note that the wave peak levels are only 1-2 orders above noise for the lower ppc/s run shown in Figure 10 and must thus be treated with care, including the growth rates derived from them. However, in general,
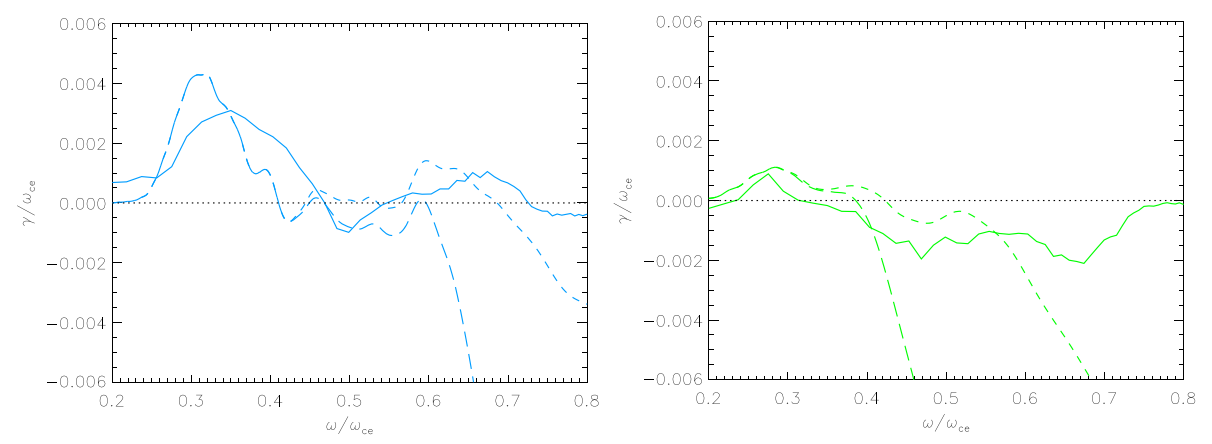

Figure 9. (left) (Blue) at $0.02 \mathrm{~s}$, (right) (green) $0.045 \mathrm{~s}$ (colors match those in Figure 5). In both panels, the solid line is the growth rate derived from the wave spectra, the short dash, the predicted growth using the hot electron distribution only, and the long dash, the predicted growth from hot and warm electron components combined. The predictions are smoothed in omega. 

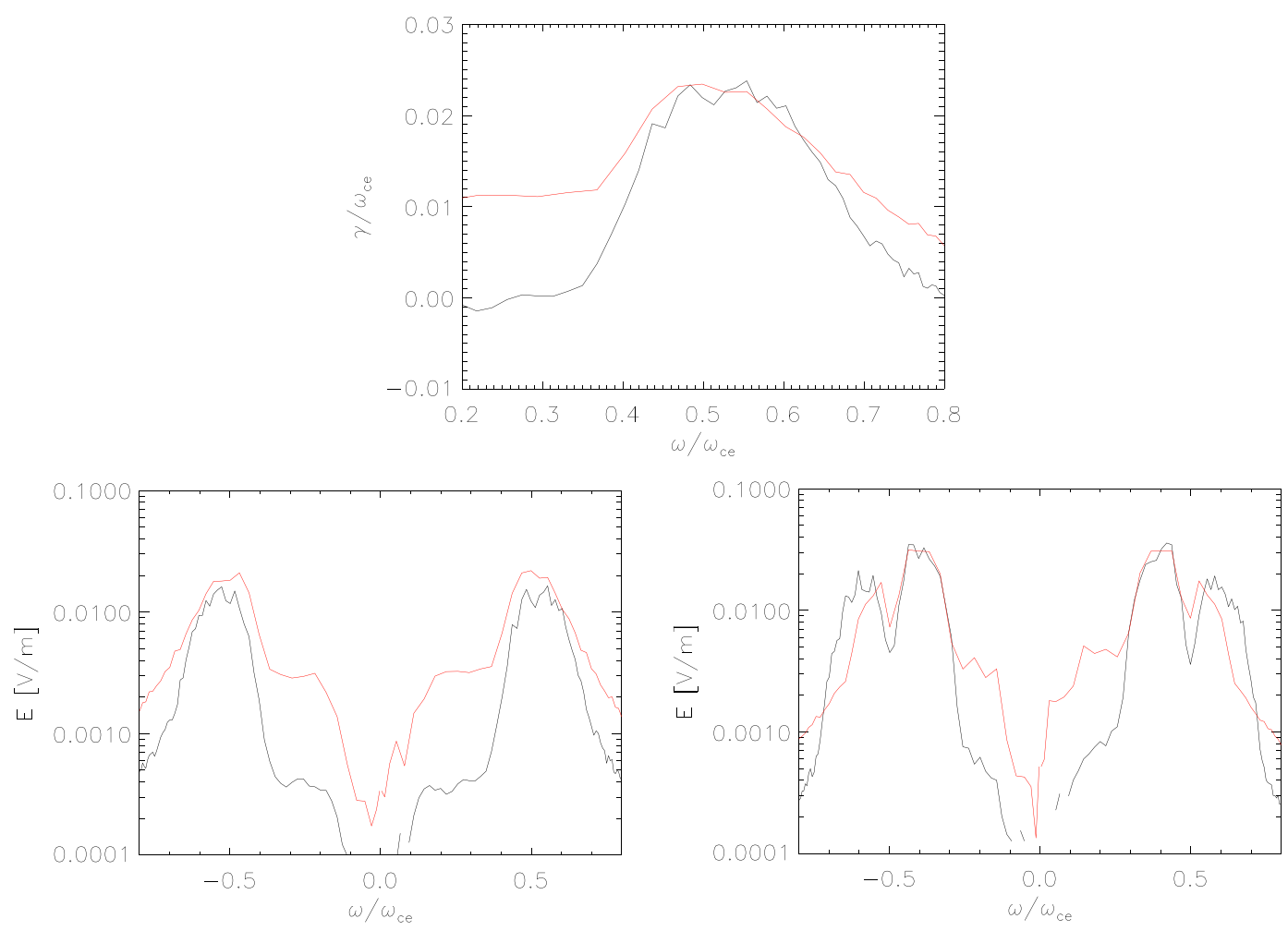

Figure 10. Convergence testing based on the $E_{y}$ field component. All panels use parameters from the "typical" simulation, M1. The red lines show $512 \times 256$ at $150 \mathrm{ppc} / \mathrm{s}$ and the black $1024 \times 512$ at $500 \mathrm{ppc} / \mathrm{s}$. (top) The growth rates calculated from the change in electric field spectra over $t=0$ to $t=0.006 \mathrm{~s}$. (bottom) The wave spectra at $t=0.003 \mathrm{~s}$ (left) and $t=0.027 \mathrm{~s}$ (right).

by $500 \mathrm{ppc} / \mathrm{s}$, our numerical experiment results show that good convergence and no qualitative changes in behavior are seen as particle number is increased.

\subsection{Angular Distribution of Wave Power and 1-D Simulation Comparisons}

Figure 11 shows the 2-D $k_{x} k_{y}$ distributions for the $E_{y}$ component of whistler mode waves at $t=0.045 \mathrm{~s}$. This is obtained by performing the 3-D FFT in space and time and then extracting a band around the whistler mode dispersion curve, with small (10\% local frequency) width in the $\omega$ direction and summing the result in omega. The two-peaked distribution is clearly evident. Moreover, the upper peak (above $0.5 \omega_{\text {ce }}$ ) shows clear evidence of angular modification, with wave power concentrated around 5 to $20^{\circ}$ off axis and reduced on axis. At these wave numbers we have an angular resolution of around $5^{\circ}$ in the lower band and $3^{\circ}$ in the upper band. The angular features strongly suggest an angular dependence of the warm plasma damping effect which acts to

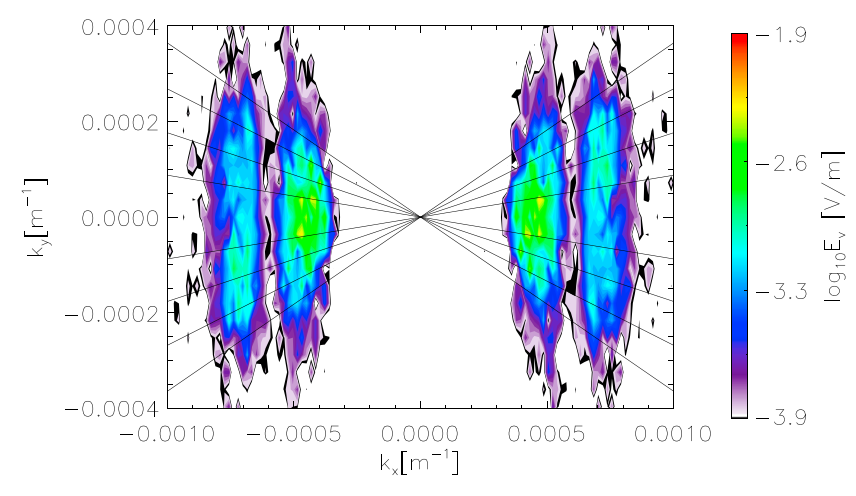

Figure 11. The angular distribution of whistler mode power at $t=0.05 \mathrm{~s}$. The solid lines show $5,10,15$, and $20^{\circ}$ angles. absorb waves in this region and/or the hot plasma growth rates.

Further evidence of the angular dependence is provided by repeating the simulations in 1-D with the same typical parameters as above. In this case waves cannot propagate at an angle, or more accurately, there can be no $y$ variation of the $E$ and $B$ fields.

Figure 12 shows the 1-D and 2-D spectra at $0.045 \mathrm{~s}$. Based on Figure 3 we expect broadband growth between 0.3 and $0.7 \omega_{\text {ce, }}$ with a peak around $0.5 \omega_{\text {ce }}$ and roughly Gaussian shape. 


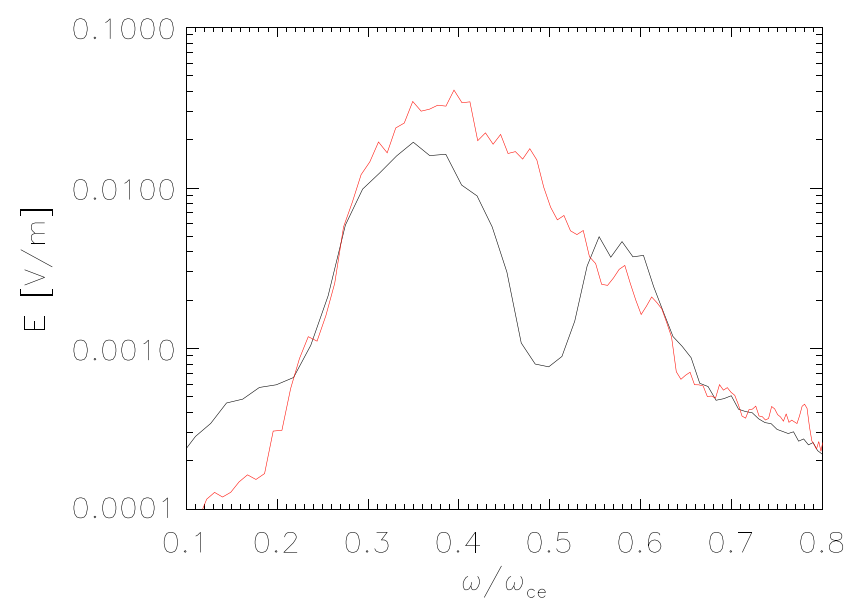

Figure 12. One- and two-dimensional simulations using both warm and hot plasma components, at $t=0.045 \mathrm{~s}$.
The 1-D case in fact matches this rather well. There is some growth down to $0.2 \omega_{\text {ce }}$ and significant asymmetry around the peak, but the general prediction holds. The asymmetry, in particular, is easily explained by damping from the rapidly evolving warm plasma component. The clear dropout seen in 2-D is entirely absent from the 1-D numerical experiment. In agreement with this, there is no kink feature in the hot electron distribution. This strongly suggests that the angular extent or scattering of the whistler mode waves is crucial to forming the dropout.

\subsection{Runs With Other Parameters}

Figure 1 showed the linear theory-predicted growth rates for a variety of parameters, including those used by Fu et al. [2014] (black line) and our typical simulation (M1, purple). It is clear that small variations in the hot and warm component parameters lead to significant changes in predicted growth rate. In the "typical" run of section 4.1, the gap feature was clearly associated with the kink in hot electron distribution, which itself appeared to be associated with the location of predicted peak wave growth. For cases M4 and M5 the gap is located at a lower frequency, 0.2 to $0.3 \Omega_{\text {ce }}$ and so can be identified specifically as the $n=-1$ resonance, as noted in 4.1 .

Figure 13 shows the $0.02 \mathrm{~s}$ spectra for five numerical experiments; the experiment described in section 4.1 (M1, purple line) and four more with parameters as listed in Table 1. It also shows the derivatives of the parallel component of hot electron distribution. All five show evidence of a kink feature at $p \simeq 0.15 m_{e} / c$ which resonantly corresponds to $\omega=0.5 \omega_{\text {ce }}$. The red and orange lines, for M4 and M5 (corresponding to the tenuous, more anisotropic simulations) show this very weakly and also show a kink feature at around $p=0.4$ to $0.55 m_{e} / c$, i.e., around $\omega=0.15 \omega_{\text {ce }}$ to $0.22 \omega_{\text {ce }}$.

All five cases show a clear gap formation in the wave spectra. The gap appears roughly where peak growth is expected from the initial conditions of experiments M1 and M3-M5, shown in Figure 14. In experiments $M 1$ and $M 3-M 5$, the gap also neatly corresponds to the kink feature in the distribution. Case M2 shows a gap feature at $0.5 \omega_{\text {ce }}$ but also shows unexpected growth over the entire upper band, from $0.4 \omega_{\text {ce }}$ upward. This may be connected with the warm plasma component, which we saw earlier and in Figure 14 strongly affects the high-frequency prediction above $0.7 \omega_{\text {ce }}$.
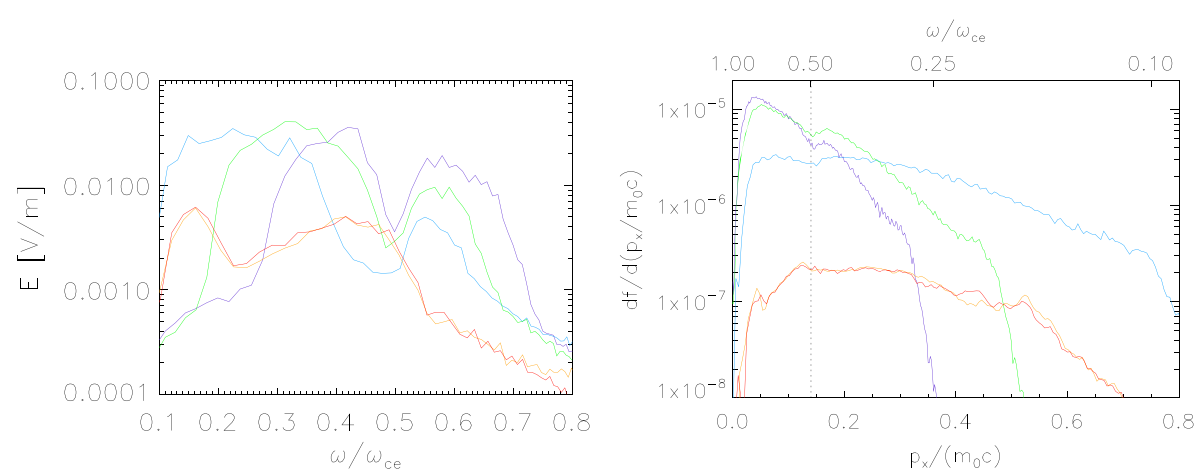

Figure 13. Wave spectra and hot component parallel derivatives for the five simulations at $t=0.025 \mathrm{~s}$. Colors are as in Figure 1: M1 is shown in purple, M2 in blue, M3 in green, M4 in orange, and M5 in red. 

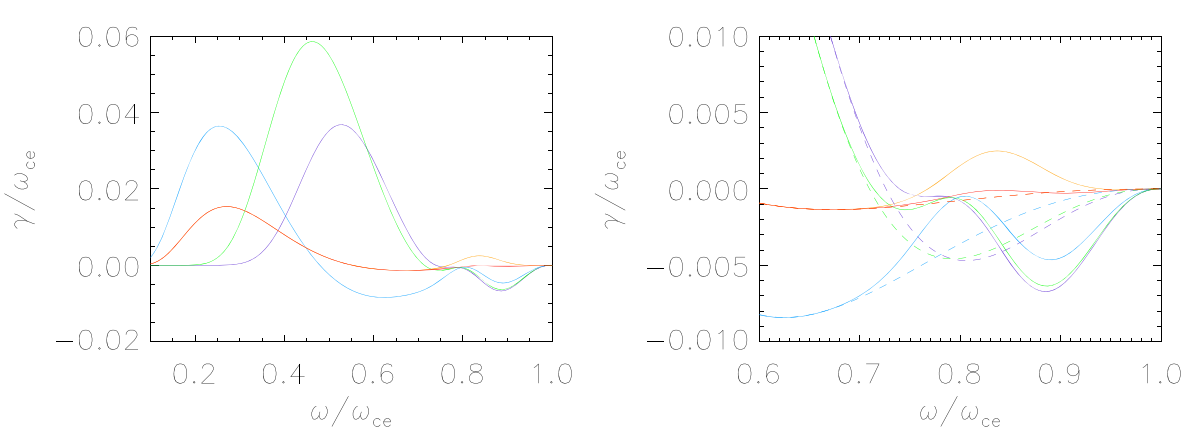

Figure 14. (left) Growth rates from linear theory applied to the initial distributions for the five runs and (right) the high-frequency part with (solid) and without (dashed) the warm plasma component contribution included. Colors are as in the previous figure.

\section{Discussion and Summary}

The preceding results show that for a range of plausible magnetospheric hot plasma parameters, a gap feature arises self-consistently in whistler mode spectra. For most numerical experiments we have performed, this gap lies at approximately $0.5 \omega_{\mathrm{ce}}$, as often observed in naturally occurring whistler mode waves in the magnetosphere [Burtis and Helliwell, 1969; Santolík et al., 2004; Santolik et al., 2008; Taubenschuss et al., 2014]. One of our simulations, $M 2$, did not show a convincing gap feature, as its upper band is mostly absent.

Based on the 1-D and 2-D simulations and the analysis of distribution functions, a picture arises. The waves grow initially as expected by linear growth theory for a hot anisotropic Maxwellian. For the simulation discussed in detail in section 4.1 , the peak wave growth is then at $0.5 \omega_{\text {ce }}$. In response to these waves, a region of reduced $\partial f / \partial p_{\|}$quickly forms in the parallel direction of the electron distribution function over a small region corresponding to the phase velocity of the waves with largest growth rates. This shoulder feature does not form in one-dimensional simulations, but these constrain $\mathbf{k} \times \mathbf{B}=0$, and so there is no resulting $E_{\|}$to modify the electrons in the parallel direction. In our simulations, we find that at least two spatial dimensions are necessary to observe the modification of $f$ required to explain the formation of a whistler mode spectral gap, as suggested by Katoh and Omura [2016]. Fourier analysis of the $E_{x}$ (parallel to $\mathbf{B}$ ) component of wave power shows a weak signal, centered around $20^{\circ}$ off axis, and generally more oblique for the upper band of waves. It appears that the oblique wave numbers, even though they initially have weaker growth rates than those with $k_{\perp}=0$, are critical to the process, providing $E_{\|}$required to modify the distribution function in the parallel direction. The action of oblique whistler mode waves is a subject of growing importance in magnetospheric physics [see, e.g., Artemyev et al., 2016, and references therein], and our results show that they are crucially important for the evolution of the anisotropy-driven whistler mode wave instability.

The modification of the distribution may be interpreted as an inverse Landau-damping-type effect, where waves are produced toward the upper end of the "dropout" feature, around $0.6 \omega_{\text {ce }}$ for the typical case, resonant with electrons in and below the kink in the hot electron distribution. Waves are driven off axis by a few degrees, reaching around $10-15^{\circ}$ over time and are absorbed by different particles at a new, lower, $v_{\|}$. The net effect of processes like this is to transfer energy from the slower to the faster particles, with the waves as a conduit.

The key common aspect of our simulations is the formation of a plateau in the distribution function in the parallel momentum direction. This plateau forms as a result of wave-particle interactions and was not part of the initial conditions. In situ observations have repeatedly demonstrated that anisotropy in electron distributions can be energy dependent [e.g., Li et al., 2011a; Ni et al., 2011 b; Fu et al., 2014]. Plateau formation in the parallel direction is one way to decrease the anisotropy of a $T_{\|}<T_{\perp}$ distribution over a small energy range. Multiple examples of plateau formation over the energy range $\sim 2-10 \mathrm{keV}$ (which corresponds to $\sim 0.1-0.15 \mathrm{c}$ as seen in our simulations) are shown by Li et al. [2016], although their analysis concerns the Landau resonance plateau at lower energies. Agapitov et al. [2015] also studied plateau formation at around 1-20 keV in the measured electron parallel velocity distribution, attributed to Landau resonance with observed oblique chorus waves. Agapitov et al. [2016] suggested that any sufficiently anisotropic initial distribution could quickly relax to form such a plateau and further showed that parallel acceleration of low-energy electrons by the electric field of very oblique waves up to the energy range for cyclotron resonance with parallel waves is very efficient and 
may result in a quick suppression of the temperature anisotropy necessary for parallel chorus wave growth. However, these studies mainly focused on the effects of very oblique waves and did not consider effects on the $0.5 \omega_{\text {ce }}$ gap. Some authors have used these features in the observed electron distribution as the initial point of their instability analysis. We show that the plateau could be a natural consequence of whistler mode wave growth from an original Bi-Maxwellian type of distribution. Once the plateau has formed, linear theory can once again be used to estimate the resulting growth rates due to the modified distribution function (e.g., Figure 7), demonstrating that growth is suppressed near $0.5 \omega_{\text {ce }}$ and enhanced above and below this frequency. Subsequent wave growth from the more structured anisotropic distribution results in the banded structure of whistler mode emission that is frequently observed.

These changes in growth rates occur very rapidly, over tens of wave periods as the distribution function remodels. As these simulations are constrained for computational reasons to run as an initial-value-type problem, we expect the evolution to proceed all at once. In reality, energetic electron injections across $1-100 \mathrm{keV}$ from the nightside that drift into the morning sector [Asnes et al., 2005; Tao et al., 2011] are more gradual, and the distribution remodeling may be continually reformed by drifting electrons. However, such a long, slow injection requires greatly increased simulation particle numbers to adequately reproduce weak waves. Additionally, repeated injections of particles into already disturbed plasma will change the early growth behavior. The simulations presented here have thermal noise (due to random motions and the restricted pseudo-particle number) which is orders-of-magnitude larger than true thermal wave levels, but still 1 to 2 orders of magnitude lower than the wave saturation levels. Strongly disturbed plasma will speed up initial growth: if this were to reach the true nonlinear regime, the explanation of Omura et al. [2008, 2009] would apply.

More realistic first-principles simulations pose many computational challenges, but we suggest that further insight may be gained from running simulations with rapidly growing waves, similar to those documented in this paper. Future work will include some kind of source term that mimics the remodeling of the distribution function as a result of electron drift around the Earth. The effects of variation of magnetic field strength with latitude and the refractive effects this has on the waves could also be usefully explored, although a simulation domain large enough to do this is very demanding.

What is remarkable from our analysis is that the predictions of growth using linear (small wave amplitude) theory still appear to give accurate growth rates throughout the evolution of the electron distribution function. It is only important to get the details of the distribution function correct. The subtle changes in $f\left(p_{\|}\right)$ yield large changes in the predicted growth rate $\gamma$, a phenomenon we will investigate further in future work. Further, as the distribution remodeling appears to be due to the rapid initial wave growth, we would expect lower or wider band wave growth rates not to produce this feature and any other thermalization processes to suppress it. This offers an immediate explanation for the not uncommon absence of the gap feature.

In summary, we have shown that self-consistent simulations can produce a spectral gap for whistler mode waves for a broad range of hot electron parameters. The central frequency of this gap depends on plasma parameters and often occurs at $0.5 \omega_{\text {ce }}$. The effect is seen to be inherently two-dimensional and to require good reproduction of small local changes in the distribution function, making it very difficult to capture numerically. Future work will consider higher particle number simulations to improve reproduction of the effect and also address the differences between the initial-value setup and more physical continuous injection of fast particles.

\section{References}

Agapitov, O., A. Artemyev, V. Krasnoselskikh, Y. V. Khotyaintsev, D. Mourenas, H. Breuillard, M. Balikhin, and G. Rolland (2013), Statistics of whistler mode waves in the outer radiation belt: Cluster STAFF-SA measurements, J. Geophys. Res. Space Physics, 118, 3407-3420, doi:10.1002/jgra.50312.

Agapitov, O. V., A. V. Artemyev, D. Mourenas, F. S. Mozer, and V. Krasnoselskikh (2015), Nonlinear local parallel acceleration of electrons through Landau trapping by oblique whistler mode waves in the outer radiation belt, Geophys. Res. Lett., 42, 10,140-10,149, doi:10.1002/2015GL066887.

Agapitov, O. V., D. Mourenas, A. V. Artemyev, and F. S. Mozer (2016), Exclusion principle for very oblique and parallel lower band chorus waves, Geophys. Res. Lett., 43, 11,112-11,120, doi:10.1002/2016GL071250.

Arber, T. D., et al. (2015), Contemporary particle-in-cell approach to laser-plasma modelling, Plasma Phys. Controlled Fusion, 57(11), 1-26. Artemyev, A., O. Agapitov, D. Mourenas, V. Krasnoselskikh, V. Shastun, and F. Mozer (2016), Oblique whistler-mode waves in the Earth's inner magnetosphere: Energy distribution, origins, and role in radiation belt dynamics, Space Sci. Rev., 200, 261-355, doi:10.1007/s11214-016-0252-5. 
Artemyev, A. V., V. V. Krasnoselskikh, O. V. Agapitov, D. Mourenas, and G. Rolland (2012), Non-diffusive resonant acceleration of electrons in the radiation belts, Phys. Plasmas, 19(12), 122901, doi:10.1063/1.4769726.

Artemyev, A. V., O. V. Agapitov, D. Mourenas, V. V. Krasnoselskikh, and F. S. Mozer (2015), Wave energy budget analysis in the Earth's radiation belts uncovers a missing energy, Nat. Commun., 6, 8143, doi:10.1038/ncomms8143.

Asnes, A., R. W. Friedel, J. Stadsnes, M. Thomsen, N. Østgaard, and T. Cayton (2005), Statistical pitch angle properties of substorm-injected electron clouds and their relation to dawnside energetic electron precipitation, J. Geophys. Res., 110, A05207, doi:10.1029/2004JA010838.

Bell, T. F., U. S. Inan, N. Haque, and J. S. Pickett (2009), Source regions of banded chorus, Geophys. Res. Lett., 36, L11101, doi:10.1029/2009GL037629.

Birdsall, C., and A. Langdon (2004), Plasma Physics via Computer Simulation, Series in Plasma Physics, Taylor and Francis, Abingdon, U. K.

Bortnik, J., U. S. Inan, and T. F. Bell (2006), Landau damping and resultant unidirectional propagation of chorus waves, Geophys. Res. Lett., 33, L03102, doi:10.1029/2005GL024553.

Bunch, N. L., M. Spasojevic, and Y. Y. Shprits (2011), On the latitudinal extent of chorus emissions as observed by the polar plasma wave instrument, J. Geophys. Res., 116, A04204, doi:10.1029/2010JA016181.

Bunch, N. L., M. Spasojevic, and Y. Y. Shprits (2012), Off-equatorial chorus occurrence and wave amplitude distributions as observed by the polar plasma wave instrument, J. Geophys. Res., 117, A04205, doi:10.1029/2011JA017228.

Burtis, W., and R. Helliwell (1976), Magnetospheric chorus: Occurrence patterns and normalized frequency, Planet. Space Sci., 24(11), 1007-1024, doi:10.1016/0032-0633(76)90119-7.

Burtis, W. J., and R. A. Helliwell (1969), Banded chorus - A new type of VLF radiation observed in the magnetosphere by OGO 1 and OGO 3 , J. Geophys. Res., 74(11), 3002-3010, doi:10.1029/JA074i011p03002.

Cornilleau-Wehrlin, N., R. Gendrin, F. Lefeuvre, M. Parrot, R. Grard, D. Jones, A. Bahnsen, E. Ungstrup, and W. Gibbons (1978), VLF electromagnetic waves observed onboard GEOS-1, Space Sci. Rev., 22(4), 371-382, doi:10.1007/BF00210874.

Coroniti, F. V., F. L. Scarf, C. F. Kennel, and W. S. Kurth (1984), Analysis of chorus emissions at Jupiter, J. Geophys. Res., 89(A6), 3801-3820, doi:10.1029/JA089iA06p03801.

Denton, M. H., J. E. Borovsky, and T. E. Cayton (2010), A density-temperature description of the outer electron radiation belt during geomagnetic storms, J. Geophys. Res., 115, A01208, doi:10.1029/2009JA014183.

$\mathrm{Fu}, \mathrm{X}$., et al. (2014), Whistler anisotropy instabilities as the source of banded chorus: Van Allen Probes observations and particle-in-cell simulations, J. Geophys. Res. Space Physics, 119, 8288-8298, doi:10.1002/2014JA020364.

Gannon, J. L., X. Li, and D. Heynderickx (2007), Pitch angle distribution analysis of radiation belt electrons based on Combined Release and Radiation Effects Satellite Medium Electrons A data, J. Geophys. Res., 112, A05212, doi:10.1029/2005JA011565.

Gao, X., D. Mourenas, W. Li, A. V. Artemyev, Q. Lu, X. Tao, and S. Wang (2016), Observational evidence of generation mechanisms for very oblique lower band chorus using THEMIS waveform data, J. Geophys. Res. Space Physics, 121, 6732-6748, doi:10.1002/2016JA022915.

Haque, N., M. Spasojevic, O. Santolik, and U. S. Inan (2010), Wave normal angles of magnetospheric chorus emissions observed on the Polar spacecraft, J. Geophys. Res., 115, A00F07, doi:10.1029/2009JA014717.

Hartley, D. P., C. A. Kletzing, W. S. Kurth, S. R. Bounds, T. F. Averkamp, G. B. Hospodarsky, J. R. Wygant, J. W. Bonnell, O. Santolík, and C. E. J. Watt (2016), Using the cold plasma dispersion relation and whistler mode waves to quantify the antenna sheath impedance of the Van Allen probes EFW instrument, J. Geophys. Res. Space Physics, 121, 4590-4606, doi:10.1002/2016JA022501.

Horne, R., et al. (2005), Wave acceleration of electrons in the Van Allen radiation belts, Nature, 437, 227-230, doi:10.1038/nature03939.

Katoh, Y., and Y. Omura (2016), Electron hybrid code simulation of whistler-mode chorus generation with real parameters in the Earth's inner magnetosphere, Earth Planets Space, 68, 192, doi:10.1186/s40623-016-0568-0.

Kurita, S., Y. Katoh, Y. Omura, V. Angelopoulos, C. M. Cully, O. Le Contel, and H. Misawa (2012), Themis observation of chorus elements without a gap at half the gyrofrequency, J. Geophys. Res., 117, A11223, doi:10.1029/2012JA018076.

$\mathrm{Li}$, W., J. Bortnik, R. M. Thorne, and V. Angelopoulos (2011a), Global distribution of wave amplitudes and wave normal angles of chorus waves using THEMIS wave observations, J. Geophys. Res., 116, A12205, doi:10.1029/2011 JA017035.

Li, W., J. Bortnik, R. M. Thorne, Y. Nishimura, V. Angelopoulos, and L. Chen (2011b), Modulation of whistler mode chorus waves: 2. Role of density variations, J. Geophys. Res., 116, A06206, doi:10.1029/2010JA016313.

Li, W., R. M. Thorne, J. Bortnik, Y. Nishimura, and V. Angelopoulos (2011c), Modulation of whistler mode chorus waves: 1. Role of compressional Pc4-5 pulsations, J. Geophys. Res., 116, A06205, doi:10.1029/2010JA016312.

Li, W., et al. (2016), Unraveling the excitation mechanisms of highly oblique lower band chorus waves, Geophys. Res. Lett., 43, 8867-8875, doi:10.1002/2016GL070386.

Li, W., R. M. Thorne, J. Bortnik, X. Tao, and V. Angelopoulos (2012), Characteristics of hiss-like and discrete whistler-mode emissions, Geophys. Res. Lett., 39, L18106, doi:10.1029/2012GL053206.

Li, W., J. Bortnik, R. M. Thorne, C. M. Cully, L. Chen, V. Angelopoulos, Y. Nishimura, J. B. Tao, J. W. Bonnell, and O. LeContel (2013), Characteristics of the Poynting flux and wave normal vectors of whistler-mode waves observed on THEMIS, J. Geophys. Res. Space Physics, 118, 1461-1471, doi:10.1002/jgra.50176.

Liu, K., S. P. Gary, and D. Winske (2011), Excitation of banded whistler waves in the magnetosphere, Geophys. Res. Lett., 38, L14108, doi:10.1029/2011GL048375.

Meredith, N. P., A. D. Johnstone, S. Szita, R. B. Horne, and R. R. Anderson (1999), “Pancake” electron distributions in the outer radiation belts, J. Geophys. Res., 104, 12,431-12,444, doi:10.1029/1998JA900083.

Meredith, N. P., R. B. Horne, and R. R. Anderson (2001), Substorm dependence of chorus amplitudes: Implications for the acceleration of electrons to relativistic energies, J. Geophys. Res., 106(A7), 13,165-13,178, doi:10.1029/2000JA900156.

Meredith, N. P., R. B. Horne, A. Sicard-Piet, D. Boscher, K. H. Yearby, W. Li, and R. M. Thorne (2012), Global model of lower band and upper band chorus from multiple satellite observations, J. Geophys. Res., 117, A10225, doi:10.1029/2012JA017978.

Ni, B., R. M. Thorne, N. P. Meredith, Y. Y. Shprits, and R. B. Horne (2011a), Diffuse auroral scattering by whistler mode chorus waves: Dependence on wave normal angle distribution, J. Geophys. Res., 116, A10207, doi:10.1029/2011JA016517.

Ni, B., R. M. Thorne, N. P. Meredith, R. B. Horne, and Y. Y. Shprits (2011b), Resonant scattering of plasma sheet electrons leading to diffuse auroral precipitation: 2. Evaluation for whistler mode chorus waves, J. Geophys. Res., 116, A04219, doi:10.1029/2010JA016233.

Nishimura, Y., et al. (2013), Structures of dayside whistler-mode waves deduced from conjugate diffuse aurora, J. Geophys. Res. Space Physics, 118, 664-673, doi:10.1029/2012JA018242.

Omura, Y., Y. Katoh, and D. Summers (2008), Theory and simulation of the generation of whistler-mode chorus, J. Geophys. Res., 113, A04223, doi:10.1029/2007JA012622.

Omura, Y., M. Hikishima, Y. Katoh, D. Summers, and S. Yagitani (2009), Nonlinear mechanisms of lower-band and upper-band VLF chorus emissions in the magnetosphere, J. Geophys. Res., 114, A07217, doi:10.1029/2009JA014206. 
Santolík, O., D. A. Gurnett, and J. S. Pickett (2004), Multipoint investigation of the source region of storm-time chorus, Ann. Geophys., 22(7), 2555-2563, doi:10.5194/angeo-22-2555-2004.

Santolik, O., E. Macusova, E. E. Titova, B. V. Kozelov, D. A. Gurnett, J. S. Pickett, V. Y. Trakhtengerts, and A. G. Demekhov (2008), Frequencies of wave packets of whistler-mode chorus inside its source region: A case study, Ann. Geophys., 26(6), 1665-1670, doi:10.5194/angeo-26-1665-2008.

Sigsbee, K., J. D. Menietti, O. Santolík, and J. S. Pickett (2010), Locations of chorus emissions observed by the polar plasma wave instrument, J. Geophys. Res., 115, A00F12, doi:10.1029/2009JA014579.

Spasojevic, M., and U. S. Inan (2010), Drivers of chorus in the outer dayside magnetosphere, J. Geophys. Res., 115, A00F09, doi:10.1029/2009JA014452.

Tao, X., R. M. Thorne, W. Li, B. Ni, N. P. Meredith, and R. B. Horne (2011), Evolution of electron pitch angle distributions following injection from the plasma sheet, J. Geophys. Res., 116, A04229, doi:10.1029/2010JA016245.

Taubenschuss, U., Y. V. Khotyaintsev, O. Santolík, A. Vaivads, C. M. Cully, O. L. Contel, and V. Angelopoulos (2014), Wave normal angles of whistler mode chorus rising and falling tones, J. Geophys. Res. Space Physics, 119, 9567-9578, doi:10.1002/2014JA020575.

Taubenschuss, U., O. Santolík, D. B. Graham, H. Fu, Y. V. Khotyaintsev, and O. Le Contel (2015), Different types of whistler mode chorus in the equatorial source region, Geophys. Res. Lett., 42, 8271-8279, doi:10.1002/2015GL066004.

Taubenschuss, U., O. Santolik, H. Breuillard, W. Li, and O. Le Contel (2016), Poynting vector and wave vector directions of equatorial chorus, J. Geophys. Res. Space Physics, 121, 11,912-11,928, doi:10.1002/2016JA023389.

Thorne, R. M., B. Ni, X. Tao, R. B. Horne, and N. P. Meredith (2010), Scattering by chorus waves as the dominant cause of diffuse auroral precipitation, Nature, 467, 943-946, doi:10.1038/nature09467.

Thorne, R. M., et al. (2013), Rapid local acceleration of relativistic radiation-belt electrons by magnetospheric chorus, Nature, 504, 411-414, doi:10.1038/nature12889.

Treumann, R. A., and W. Baumjohann (1997), Advanced Space Plasma Physics, Imperial College Press, London.

Tsurutani, B. T., and E. J. Smith (1977), Two types of magnetospheric ELF chorus and their substorm dependences, J. Geophys. Res., 82(32), 5112-5128, doi:10.1029/JA082i032p05112.

Villasenor, J., and O. Buneman (1992), Rigorous charge conservation for local electromagnetic field solvers, Comput. Phys. Commun., 69(2-3), 306-316, doi:10.1016/0010-4655(92)90169-Y.

Watt, C. E. J., A. W. Degeling, R. Rankin, K. R. Murphy, I. J. Rae, and H. J. Singer (2011), Ultralow-frequency modulation of whistler-mode wave growth, J. Geophys. Res., 116, A10209, doi:10.1029/2011JA016730.

Watt, C. E. J., R. Rankin, and A. W. Degeling (2012), Whistler mode wave growth and propagation in the prenoon magnetosphere, J. Geophys. Res., 117, A06205, doi:10.1029/2012JA017765.

Wrenn, G. L., J. F. E. Johnson, and J. J. Sojka (1979), Stable 'pancake' distributions of low-energy electrons in the plasma trough, Nature, 279, $512-514$.

Xiao, F., R. M. Thorne, and D. Summers (1998), Instability of electromagnetic R-mode waves in a relativistic plasma, Phys. Plasmas, 5 , 2489-2497, doi:10.1063/1.872932. 